\title{
Prognosis and Clinicopathology of CXCR4 in Colorectal Cancer Patients: a Meta-analysis
}

\author{
Lu-Ning Li ${ }^{1}$, Kai-Tong Jiang ${ }^{1}$, Peng Tan ${ }^{2}$, Ai-Hua Wang ${ }^{1}$, Qing-Yin Kong ${ }^{1}$, Cui- \\ Yue Wang ${ }^{1}$, Hua-Rong Lü ${ }^{1}$, Jing Wang ${ }^{3 *}$
}

\begin{abstract}
The chemokine receptor 4 (CXCR4) has been widely used in diagnosis and prognosis of colorectal cancer (CRC). However, there is no current consensus on the impact of CXCR4 on CRC patients. The purpose of this study was to evaluate the prognostic and clinicopathological importance of CXCR4 in CRC patients. Databases, such as PubMed, Cochrane library, CBM and EMBASE updated to 2014 were searched to include eligible articles. We analysed correlations between CXCR4 expression and clinicopathological features and overall survival (OS). A total of 1, 055 CRC patients from twelve studies were included in the study. The pooled odds ratios (ORs) which indicated CXCR4 expression was likely to be associated with $\mathrm{TNM}$ stage $(\mathrm{OR}=0.43, \mathrm{CI}=0.34-0.55$, $P<0.00001)$, lymph node status $(O R=2.23, C I=1.23-4.05, P=0.008)$ and vascular invasion $(O R=2.21, C I=1.11-4.39$, $P=0.02)$. Poor overall survival of CRC cancer was found to be significantly related to CXCR4 overexpression (hazard ratio $(\mathrm{HR})$ 1.36 $\mathrm{CI}=1.17-1.59, P<0.0001)$, whereas combined ORs revealed that $\mathrm{CXCR} 4$ expression had no correlation with gender or differentiation. Based on the published studies, CXCR4 overexpression in patients with CRC indicates poor survival outcome and clinicopathological factors.
\end{abstract}

Keywords: Prognosis - clinicopathology - CXCR4 - colorectal cancer

Asian Pac J Cancer Prev, 16 (9), 4077-4080

\section{Introduction}

Colorectal cancer (CRC) is the third most common cancer in males and the second most common cancer in females worldwide and has high mortality rates (Jemal et al., 2011). CRC patients are usually treated with surgery, post-operative recurrence and metastasis remain the two most troublesome problems for prolonging patient's survival time after surgery. Therefore, it is necessary to understand the etiology and pathogenesis of canceration.

Recent studies have highlighted the role of chemokines and their receptors in cancer metastasis (Stetler et al., 2001). CXC chemokine receptor 4 (CXCR4) and its corresponding chemokine ligand play an important role in the mechanism of cancer metastasis. CXCR4 is mostly expressed in various cancers including $\mathrm{CRC}$, breast cancer, gastric cancer, esophageal cancer, prostate cancer, and ovarian cancer, and so on (Balkwill et al., 2004). The expression of CXCR4 affects the metastatic behavior of CRC cells, which is associated with poor patient prognosis. CXCR4-overexpressing cancer cells have an increased ability of migration in vitro and metastasis to other organs (Kang et al., 2003; Luker et al., 2006).

Although evidence showed that CXCR4 plays an important role in in CRC patients, there were some controversies. This meta-analysis aims to evaluate the value of CXCR4 as a prognostic and clinicopathological marker for colorectal cancer patients.

\section{Materials and Methods}

\section{Literature search strategy}

We comprehensively searched PubMed, Cochrane library, CBM and EMBASE databases for relevant articles published updated to June2014.The searching terms used were: colorectal cancer ("colorectal cancer", "colorectal carcinoma", "colorectal tumor"), CXCR4 ("CXCR4", "chemokine receptor 4"), prognosis or outcome, survival. All bibliographies were indentified in the reference lists.

\section{Criteria for inclusion and exclusion}

Studies eligible for inclusion in this meta-analysis met the following criteria in order to ensure the high quality of this article: $i$ ) All the patients with colorectal cancer diagnosed by pathology, who underwent surgical resection; $i$ i) researches revealed the relationship between CXCR4 expression and colorectal cancer clinicopathological parameters and prognosis; $i i i)$ CXCR4 expression was detected by immunohistochemistry (IHC) method or reverse transcription polymerase chain

${ }^{1}$ Department of Gastroenterology, Linyi People's Hospital, ${ }^{2}$ Internal medicine teaching and research section of Shandong Medical College, Linyi, ${ }^{3}$ Department of Hepatology, Provincial Hospital Affiliated to Shandong University, Jinan, China *For correspondence: Wjingsmile123@126.com 


\section{Lu-Ning Li et al}

reaction (RT-PCR); $i v$ ) for duplicate articles, only the most complete and/or recently published one was included and $v$ ) publications in English. The following studies were excluded: $i$ ) articles about cell lines or animals; $i i$ ) review articles without original data.

\section{Data extraction}

The following information were collected independently by 2 reviewers ( $\mathrm{Li}$ and Wang) from each publication: first author, publication time, country, number of patients enrolled, method, antibody source, dilution, as well as other related events. Disagreements between the two reviewers were settled by discussion with a third reviewer.

\section{Statistical analysis}

Analysis was performed using the Review Manager 5.1 (Cochrane Collaboration, Oxford, UK). Comparisons of dichotomous measures were performed by pooled estimates of odds ratios (ORs) as well as

their $95 \%$ CIs. $P$ value of $<0.05$ was considered to be statistically significant. Heterogeneity was tested using a chi-square test with significance being set at $P<0.10$; the total variation among studies was estimated by I square. If there was heterogeneity among studies, we used a random effect model to pool the ORs; otherwise, a fixed effect model was selected.

\section{Results}

\section{Identification of relevant studies}

The flow graph of articles selection was shown in Figure 1. At first, 116 articles were identified using the search strategy. Original data were initially screened, based on paper titles and abstracts. 98 articles not relevant to our goal were excluded. Among the 18 articles, six of those were excluded due to laboratory studies, non-original articles (review), or studies irrelevant to the current analysis. Eventually, the remaining twelve studies were included for further analysis (Joseph et al., 2005; Alessandro et al., 2006; Fukunaga et al., 2006; N Yoshitake et al., 2008; Frank et al., 2009; Shang et al., 2010; Yugang et al., 2010; Adam et al., 2012; Crescenzo et al., 2013; Tinghua et al., 2013; Ying et al., 2014; Shinichiro et al., 2014) (Figure 1).

\section{Study characteristics}

The main characteristics of the 12 studies were shown in Table 1.The total number of patients included was 1913. The studies were conducted in 4 countries (China, Taiwan, America, Italy and Japan) and published between the year 2005 and 2014. Both the IHC and RT-PCR were used to evaluate the expression of CXCR4 in colorectal cancer specimens. The most commonly used antibody was a R\&D Systems antibody against CXCR4. HR and 95\% CI were obtained from Kaplan-Meier curves. Positive CXCR4 expression was defined by IHC and RT-PCR.

\section{CXCR4 expression and clinicopathological features}

All the included studies evaluated the correlation of CXCR4 expression with gender, histological differentiation, tumor depth, vascular invasion and status of lymph node metastasis. The analysis showed that CXCR4 expression had no correlation with gender $(\mathrm{OR}=0.97, \mathrm{CI}=0.80-1.18, P=0.78$, Figure 2A) or differentiation $(\mathrm{OR}=0.55, \mathrm{CI}=0.41-0.73, P<0.0001$, Figure 2B). However, CXCR4 expression was likely to be associated with TNM stage $(\mathrm{OR}=0.43, \mathrm{CI}=0.34$ -

Table 1. Characteristics of Eligible Studies

\begin{tabular}{|c|c|c|c|c|c|c|c|}
\hline First author & Year & Origin & Cases & Method & Antibody source & Dilution & Counting method \\
\hline $\begin{array}{l}\text { Adam } \\
\text { C.Yopp }\end{array}$ & 2012 & American & 75 & IHC & R\&D & 4.208333333 & $\begin{array}{l}\text { Staining intensity score and } \\
\text { percentage of positive cells }\end{array}$ \\
\hline $\begin{array}{l}\text { Alessandro } \\
\text { Ottaiano }\end{array}$ & 2006 & Italy & 72 & IHC & $\mathrm{R} \& \mathrm{D}$ & $1: 30$ & $\begin{array}{l}\text { Staining intensity score and } \\
\text { percentage of positive cells }\end{array}$ \\
\hline $\begin{array}{l}\text { Crescenzo } \\
\text { D'Alterio }\end{array}$ & 2014 & Italy & 68 & IHC & $\mathrm{R} \& \mathrm{D}$ & 0.111111111 & \\
\hline Frank M & 2009 & American & 70 & IHC RT-PCR & $\mathrm{R} \& \mathrm{D}$ & & Staining intensity score \\
\hline Joseph Kim & 2005 & American & 139 & IHC & $\begin{array}{c}\text { antihuman } \\
\text { CXCR4antibody }\end{array}$ & 0.180555556 & \\
\hline N Yoshitake & 2008 & Japan & 60 & IHC & $\mathrm{R} \& \mathrm{D}$ & $1: 20$ & \\
\hline S. Fukunaga & 2007 & Japan & 161 & IHC & & & \\
\hline $\begin{array}{l}\text { Shang- } \\
\text { Chiung }\end{array}$ & 2010 & Taiwan & 388 & IHC & $R \& D$ & $1: 50$ & \\
\hline $\begin{array}{c}\text { Shinichiro } \\
\text { Yamada }\end{array}$ & 2014 & Japan & 16 & RT-PCR & & & \\
\hline Tinghua $\mathrm{Hu}$ & 2013 & China & 76 & & & & $\begin{array}{l}\text { Staining intensity score and } \\
\text { percentage of positive cells }\end{array}$ \\
\hline Ying Gao & 2014 & China & 720 & IHC RT-PCR & & & Staining intensity score \\
\hline Yugang Wu & 2010 & China & 68 & IHC RT-PCR & $\mathrm{R} \& \mathrm{D}$ & & $\begin{array}{l}\text { Staining intensity score and } \\
\text { percentage of positive cells }\end{array}$ \\
\hline
\end{tabular}




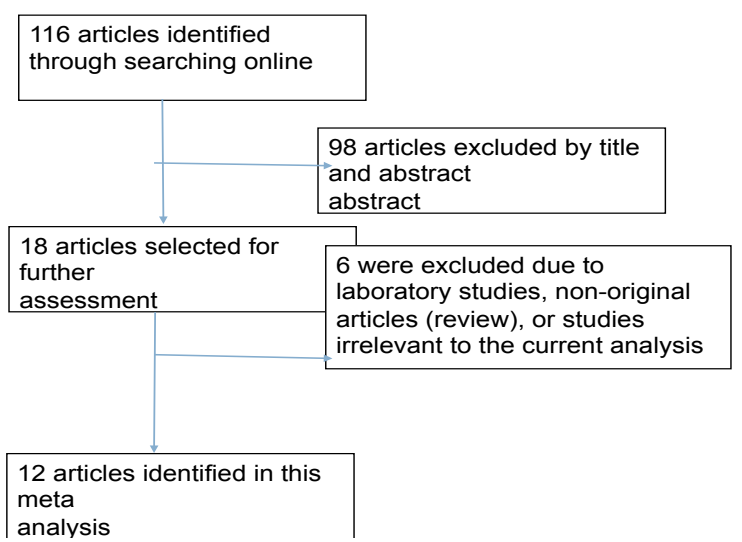

Figure 1. Flow chart for Selection of Studies

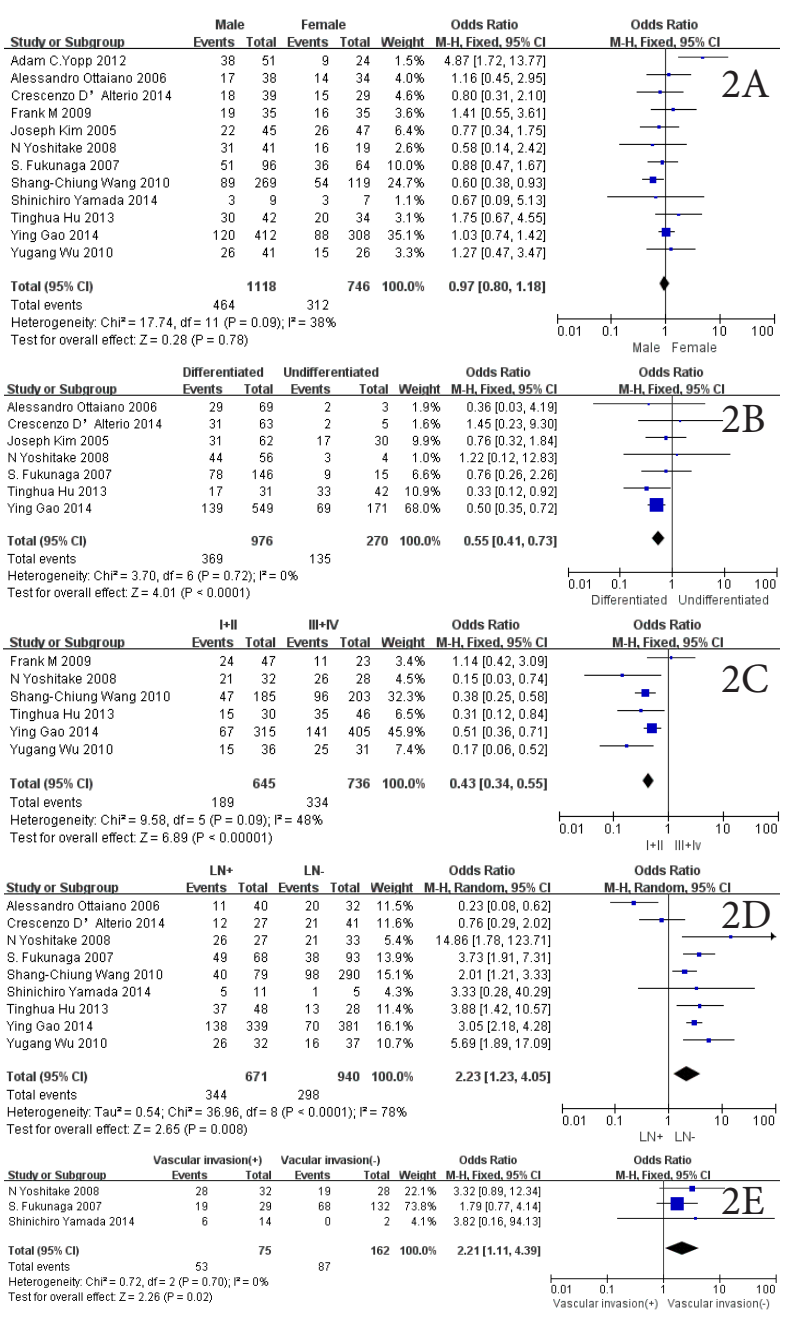

Figure 2. Forest Plots of CXCR4 Expression and the Clinicopathological Features of patients with Colorectal Cancer. A) Gender. B) Differentiation. C) TNM staging. D) Status of lymphnode. E) vascular invasion

$0.55, P<0.00001$, Figure $2 \mathrm{C}$ ) and status of lymph node $(\mathrm{OR}=2.23, \mathrm{CI}=1.23-4.05, P=0.008$, Figure 2D).

In three studies, the association of CXCR4 with vascular invasion of colorectal cancer was also investigated. It was found that CXCR4 expression was different in vascular invasion and no vascular invasion of CRC (pooled $\mathrm{OR}=2.21, \mathrm{CI}=1.11-4.39, P=0.02$, Figure $2 \mathrm{E}$ ).

The heterogeneity was observed in the analysis of CXCR 4 expression with TNM stage $\left(P=0.09 ; \mathrm{I}^{2}=48 \%\right)$ and status of lymph node $\left(P<0.0001 ; \mathrm{I}^{2}=78 \%\right)$, so a random

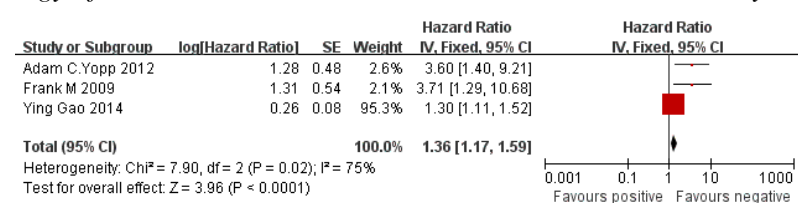

Figure 3. Forest Plot of Hazard Ratio for Overall Survival of Patients with Colorectal Cancer

effect model was used.

\section{CXCR4 as a prognostic factor for colorectal cancer}

Four out of twelve studies evaluated the relationship between OS and CXCR4 expression. The pooled HR for OS showed that overexpression of CXCR4 reduced OS in colorectal cancer. (HR=1.36, $95 \% \mathrm{CI}=1.17-1.59$, $P<0.0001$, Figure 3)

\section{Discussion}

Chemokines are a family of chemoattractant proteins that are classified depending on the arrangement of amino acids adjacent to conserved cysteine residues, which can guide the migration of cells. Some chemokines are associated with angiogenesis and cellular maturation (Kucia et al., 2004). In CRC, CXCR4 expression varies by anatomic location, with rectal primary tumors more strongly expressing CXCR4 (Schimanski et al., 2005; $\mathrm{Li}$ et al., 2015). Tumor progression is associated with intratumoral hypoxia, hypoxia upregulated CXCR4 expression in tumor cells and that the CXCR4 receptor protein level remains high at the cell membrane (Romain et al., 2014). CXCR4 expression by primary and metastatic tumors has been shown to correlate with distant metastases and overall survival (Kim et al., 2005; Ottaiano et al., 2006).

In recent years, the tumor microenvironment has become a research hotspot, which is closely related to tumorigenesis. SDF-1 and its specific receptor, CXCR4, are now receiving attention as a mechanism of cell progression and metastasis. The expression of CXCR4 has been shown to be associated with tumorigenesis and metastasis of many types of cancers (Saur et al., 2005; Hao et al., 2007; Matsusue et al., 2009). The correlation between CXCR4 expression and colorectal cancer has also been studied by many researchers. In order to determine the precise status of prognosis and clinicopathology of CXCR4 expression in colorectal cancer, we included twelve eligible studies with $1,913 \mathrm{CRC}$ patients to perform this meta-analysis.

Our meta-analysis summarized the outcomes of total 1 , 913 CRC patients from 12 individual studies, concluding that the high CXCR4 expression significantly predicted poor survival. Correlation between CXCR4 expression and differentiation, status of lymph node, and TNM staging and vascular invasion were also observed. Frank M et al. discovered that a high expression of nuclear localized CXCR4 in tumor cells is an independent predictor for poor survival for colorectal cancer patients. N Yoshitake et al. demonstrated that overexpression of CXCR4 are closely associated with $\mathrm{LN}$ metastasis and poor prognosis in patients with CRC. 
In the studies included, only three out of twelve (Frank et al., 2009; Adam et al., 2012; Ying et al., 2014) suggested statistically significant HRs for OS of elevated CXCR4 expression, and the remaining eight studies just have shown the trend of reduced survival result. An obvious association was found. if the data from these studies were combined.

There are some limitations in this stduy. Firstly, mentioned above, the number of studies and patients included is relatively small. Secondly, the different concentrations of antibody and the variable cut of definition of CXCR4 expression used in these studies might influence the final result of this meta-analysis. Thirdly, heterogeneity was found in the main analysis. Finally, not all studies offer complete results.

In conclusion, our meta-analysis showed that high CXCR4 expression was associated with poor prognosis in colorectal cancer. As a prognostic factor, CXCR4 expression can help us make further decisions on clinical therapy management.

\section{References}

Alessandro Ottaiano, Renato Franco, Annarita Aiello Talamanca, et al (2006). Overexpression of both CXC chemokine receptor 4 and vascular endothelial growth factor proteins predicts early distant relapse in stage ii-iii colorectal cancer patients. Clin Cancer Res, 12, 2795-803.

Balkwill F (2004). Cancer and the chemokine network. Nat Rev Cancer, 4, 540-50.

Crescenzo D'Alterio, Antonio Avallone, Fabiana Tatangelo, et al (2013). A prognostic model comprising pT stage, $\mathrm{N}$ status, and the chemokine receptors CXCR4 and CXCR7 powerfully predicts outcome in neoadjuvant resistant rectal cancer patients. Int J Cancer, 135, 379-90.

Frank M, Gerrit Jan, Cornelis J, et al (2009). Nuclear localization of CXCR4 determines prognosis for colorectal cancer patients. Cancer Microenvironment, 2, 1-7.

Fukunaga S, Maeda K, Noda E, et al (2006). Association between expression of vascular endothelial growth factor c, chemokine receptor CXCR4 and lymph node metastasis in colorectal cancer. Oncology, 71, 204-11.

Hao L, Zhang C, Qiu Y, et al (2007). Recombination of CXCR4, VEGF, and MMP-9 predicting lymph node metastasis in human breast cancer. Cancer Letters, 253, 34-42.

Jemal A, Bray F, Center MM, et al (2011). Global cancer statistics. CA Cancer J Clin, 61, 69-90.

Joseph Kim, Hiroya Takeuchi, Stella T. Lam, et al (2005). Chemokine Receptor CXCR4 Expression in Colorectal Cancer Patients Increases the Risk for Recurrence and for Poor Survival. J Clin Oncol, 23, 2744-53.

Kang Y, Siegel PM, Shu W, et al (2003). A multigenic program mediating breast cancer metastasis to bone. Cancer cell, 3, 537-49.

Kim J, Takeuchi H, Lam ST, et al (2005). Chemokine receptor CXCR4 expression in colorectal cancer patients increases the risk for recurrence and for poor survival. J Clin Oncol, 23, 44-53.

Kucia M, Jankowski K, Reca R, et al (2004). CXCR4-SDF-1 signalling, locomotion, chemotaxis and adhesion. $\mathrm{J} \mathrm{Mol}$ Histol, 35, 233-45.

Li XF, Guo XG, Yang YY et al., (2015). Effect of CXCR4 and CD133 co-expression on the prognosis of patients with stage II III colon cancer. Asian Pac J Cancer, 16, 1074-76
Luker KE and Luker GD (2006). Functions of CXCL12 and CXCR4 in breast cancer. Cancer Lett, 238, 30-41.

Matsusue R, Kubo H, Hisamori S, et al (2009). Hepatic stellate cells promote liver metastasis of colon cancer cells by the action of SDF-1/CXCR4 axis. Ann Surg Oncol, 16, 2645-53.

Ottaiano A, Franco R, Aiello Talamanca A, et al (2006). Overexpression of both CXC chemokine receptor 4 and vascular endothelial factor predicts early distant relapse in Stage II-III colorectal cancer patients. Clin Cancer Res, 12, 2795-803.

Romain B, Hachet-Haas M, Rohr S et al (2014). Hypoxia differentially regulated CXCR4 and CXCR7 signaling in colon cancer. Mol Cancer, 13, 58

Saur D, Seidler B, Schneider G (2005). CXCR4 expression increases liver and lung metastasis in a mouse model of pancreatic cancer. Gastroenterol, 129, 1237-50.

Schimanski CC, Schwald S, Simiantonaki N, et al (2005). Effect of chemokine receptors $\mathrm{CXCR} 4$ and CCR 7 on the metastatic behavior of human colorectal cancer. Clin Cancer Res, 11, 1743-50.

Shinichiro Yamada, Mitsuo Shimada, Tohru Utsunomiya, et al (2014). CXC receptor 4 and stromal cell-derived factor 1 in primary tumors and liver metastases of colorectal cancer.J Surg Res, 187, 107-12.

Shang-Chiung Wang, Jen-Kou Lin, Huann-Sheng Wang, et al (2010). Nuclear expression of CXCR4 is associated with advanced colorectal cancer. Int J Colorectal Dis, 25, 1185-91.

Stetler Stevenson WG, Kleiner DE Jr (2001). Molecular biology of cancer: invasionand metastasis. In: devita vt jr, hellman s, rosenberg sa, editors. cancer: principles and practice of oncology. philadelphia, PA: Lippincott Williams, 2001, 123-36.

Tinghua Hu, Yu Yao, Shuo Yu, et al (2013). Clinicopathologic significance of CXCR4 and Nrf2 in colorectal cancer. $J$ Biom Res, 27, 283-90.

Ying Gao, Chunyu Li, Min Nie, et al (2014). CXCR4 as a novel predictive biomarker for metastasis and poor prognosis in colorectal cancer. Tumor Biol, 35, 4171-5.

Yopp AC, Shia J, Butte JM, et al (2012). CXCR4 expression predicts patient outcome and recurrence patterns after hepatic resection for colorectal liver metastases. Ann Surg Oncol, 19, 339-46.

Yoshitake N, Fukui H, Yamagishi H, et al (2008). Expression of SDF-1 aand nuclear CXCR4 predicts lymph node metastasis in colorectal cancer. Br J Cancer, 98, 1682-89.

Yugang Wu, Min Jin, Huanbai Xu, et al (2010). Clinicopathologic Significance of HIF-1 $\alpha$, CXCR4, and VEGF Expression in Colon Cancer. Clin Developml Immunol. 